\title{
Scientists' perceptions of the social and political implications of their research
}

\author{
HENRY SMALl, ANN KUSHMERICK, DOUg BENSON
}

Thomson Scientific, Philadelphia, PA (USA)

\begin{abstract}
We explore an empirical approach to studying the social and political implications of science by gathering scientists' perceptions of the social impacts of their research. It was found that 78 percent of surveyed scientists from a variety of fields responding to a survey indicated that the research performed in connection with a recent highly cited paper had such implications. Health related implications were the most common, but other types of implications encountered were technological spin-offs, public understanding, economic and policy benefits. Surprisingly many scientists considered the advancement of science itself to be a social implication of their research. The relations of these implications to the field and topics of research are examined, and a mapping of implications gives an overview of the major dimensions of the social impacts of science.
\end{abstract}

\section{Introduction}

Justification for the public support of science is often couched in terms of science's benefits to society, for example its contribution to technological or economic advance, or its improvements to human health. But do scientists see their work as having relevance to social or political issues? Of course individual scientists can be motivated by a desire to help others and improve the human condition. Or they can be motivated by curiosity, a desire to seek truth, to solve puzzles, or to receive recognition from their fellow scientists. However, a specific piece of research, as for example embodied in a

Received February 12, 2007

Address for correspondence:

HENRY SMALL

Thomson Scientific, 3501 Market Street, Philadelphia, PA 19104, USA

E-mail: henry.small@thomson.com

$0138-9130 /$ US $\$ 20.00$

Copyright (C) 2007 Akadémiai Kiadó, Budapest

All rights reserved 
published paper, may or may not have achieved such an idealized or desired outcome. Our aim is to assess scientists' views on specific highly cited papers of their own, and to determine whether they perceive any social or political outcomes for that work. Focusing on specific completed work rather than generalized impressions or goals has the advantage of grounding the study in actual outcomes of research, rather than theoretical possibilities. By focusing on a sample of highly cited papers, we target this investigation to potentially significant scientific contributions.

Science policy discussions have often centered on the social warrant for science, and the positive or negative impacts of science on society. These science-on-society impacts which have intensified since the Second World War are the centerpiece of J.D. Bernal's history of science. For BERNAL, the $20^{\text {th }}$ century was the first time that "science and scientists have been involved directly and overtly in the major economic, industrial and military developments of their time." [1965, : 703] The science policy discussion was advanced by WEINBERG's classic paper [1963] in which he discussed why society should support science and what criteria should be brought to bear in such decisions. He distinguished internal and external criteria, but he regarded the external as more important. Of these, he distinguished three components: technological merit or the likelihood that a desirable technology can be created based on science; scientific merit which is essentially interdisciplinarity; and finally, the most controversial, social merit or the relevance of science to human welfare and values, such as national defense, food, and health. The problem he saw with social merit is that it depended on the value system of those setting the agenda. This is, of course, precisely the problem faced today when discussing research funding for stem cells, climate change, or nanotechnology.

Following Weinberg, the policy discussion of the social warrant of science was continued by many authors such as BROOKS [1968] with the main emphasis on technology, applied science or mission oriented science, or whether in fact there was any direct line of development from science to technology [PRICE, 1996]. This discussion has had the effect of focusing attention only on the economic payoffs and away from possible broader societal and policy implications.

Many studies have attempted to demonstrate directly how science contributes to society, as for example by seeing whether a published paper has been later used as the basis of a patented technology that achieves economic benefit [NARIN \& AL., 1997], or determining whether a medical procedure has saved lives or resulted in a measurable benefit [MURPHY \& TOPEL, 2003]. Additionally, some studies focus on the public's perception of the social implications of science [NATIONAL SCIENCE BOARD, 1993], but few examine the scientists' views on their work's social impact. An exception is the study by BARNS \& WILSON [1996] which assessed environmental scientists' perceptions of the political dimension of their field. The study by Market and Opinion Research International (MORI) published as a report in 2000 and sponsored by the Wellcome Trust [CORRADO \& AL., 2000] deserves special mention as a precursor to ours. As part 
of a larger study of scientists' attitudes toward communication with the public, MORI asked scientists to comment on the "social and ethical" implications of their research in general. Despite substantial differences in our methodologies, we will be comparing and contrasting our results with theirs.

Our approach to studying the social and political impact of science is to start from the science itself and the scientists who have produced it, and allow them to define the scope of possible impacts from their perspectives. Thus our approach does not attempt to demonstrate social benefit directly, but rather attempts to ascertain empirically how scientists themselves see the social and political implications of specific work they have performed. The advantage of this approach is that it is not based on an externally imposed value framework. It also allows a sampling of a wider range of possible social spin-offs than, for example, an investigation aimed only at the economic payoff. Our goal is to sort the authors' views into a typology of social and political implications for science that represents the spectrum of possible impact, and then to analyze the structure and disciplinary relationships of such implications into major underlying themes.

\section{Method}

Our survey was carried out as part of a broader project to collect authors' commentaries on their highly cited papers, modeled on GARFIELD's earlier work on citation classics [1981]. In contrast to Garfield's approach however, the focus is not on the most cited papers in specific fields, but rather on more recent papers that have just begun to achieve higher than expected citation rates. In general, the papers selected for this study had achieved citation counts in the top one percent within their fields in the most recent five-year period. A set of five standard questions was asked, the last of which was: "What are the social or political implications of your research?" The methodology for sampling recent highly cited papers from a number of fields has been outlined previously [SMALL, 2004]. These papers and the full interviews on which this study is based are available on the web at www.esi-topics.com under the headings "fastbreaking papers", "new hot papers", "emerging research fronts", and "fast-moving fronts".

A limitation of this type of study is that the authors' responses to the question might represent "hoped for" rather than actual social or political outcomes, or perhaps represent post-hoc justifications for work done. In the case of non-respondents, the results might reflect reluctance on the part of scientists to talk publicly. On the other hand, the advantage of this approach is that the web site is an open forum where statements are available for all to evaluate and assess in light of the actual published work. Also, the methodology allows us to sample a broader spectrum of possible social outcomes for research. This work can then be used as the basis of a more comprehensive catalogue of potential extrinsic social impacts of science, as well as to 
ascertain the most prevalent forms, how they differ across scientific fields and research topics, and how they relate to one another to form a network of social impacts.

Data collection began in October 2005 and concluded in November 2006, by which time 153 responses to the social/political question had been received out of 196 scientists who had responded to the full questionnaire for a response rate of 78 percent to that question. The remaining $22 \%$ of respondents either left the question blank or indicated that there were no such implications. This compares favorably with the 70 percent response rate obtained in the MORI study asking researchers whether there were social or ethical implications of their research. Roughly one-half of the respondents to our social/political question were based outside the USA. Since the response rate to the full questionnaire was roughly 50 percent of all researchers contacted [SMALL, 2004], the overall rate of response to the social/political question is 39 percent. Even though our sample size is small and our results therefore preliminary in nature, a majority of scientists responding to the question view their work as having some degree of social or political relevance.

Figure 1 shows the percentage by discipline of scientists responding to the social/ political implications question of those responding to the questionnaire as a whole. Discipline assignments were based on the journal in which the paper was published.

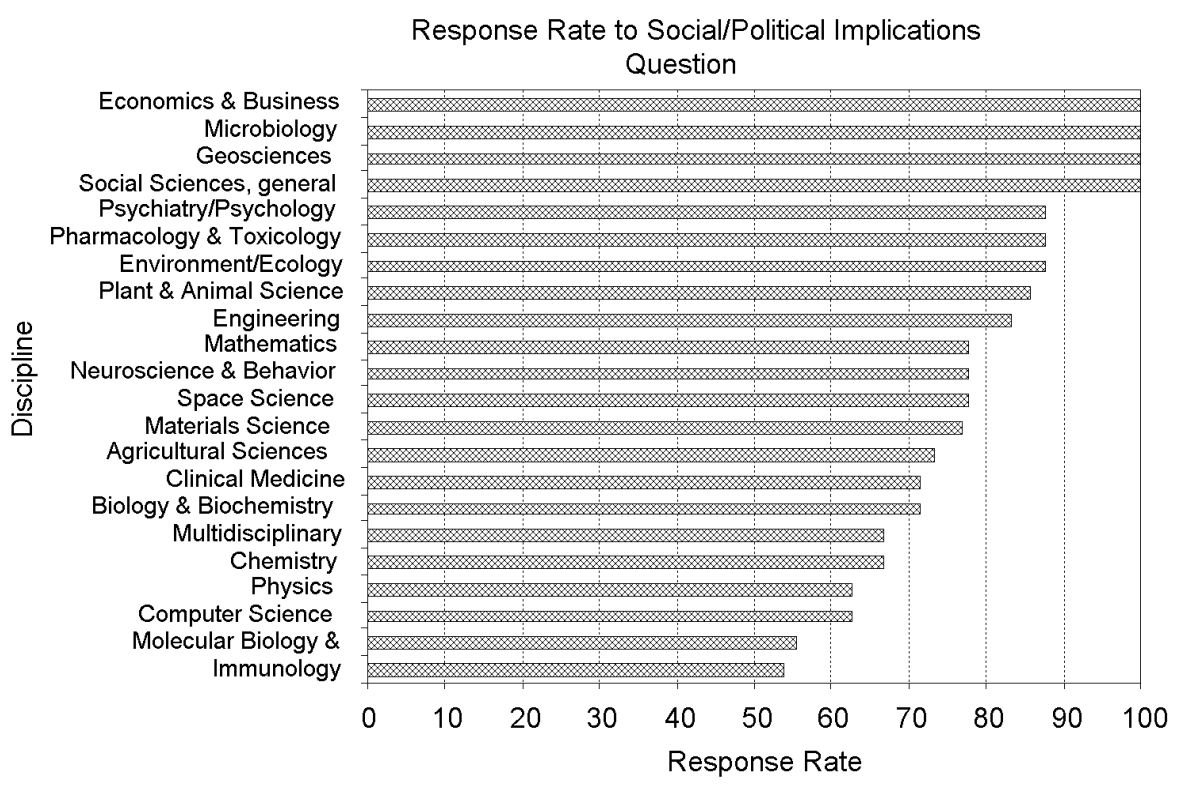

Figure 1. Response rate to social/political implications question grouped by discipline of the researcher's highly cited paper 
From Figure 1, we see that all microbiologists, geoscientists, economists, and social scientists responding to the survey reported social impacts for their work. In contrast only about $55 \%$ of the immunologists and $63 \%$ of the computer scientists indicated such impacts.

Each response was also coded by topic to obtain a finer grained breakdown than the broad journal-based categorization seen in Figure 1. The most frequent topical areas were genomics, foods, drug development, cancer, and nanotechnology. However, the topics eliciting responses ranged widely, including for example, stem cells, wireless technology, malaria, monetary theory, bio-security, ozone layer, wine contamination, human intelligence, Mars, life satisfaction, fuel cells, democracy, macular degeneration, transgenic plants, war, and pandemic influenza.

\section{Classification of social/political implications}

Based on an initial reading of the responses, a typology of social and political implications of research was constructed. The classification consisted of eight main headings, some of which had a number of subheadings (see Table 1). The main headings are general, health, environment, economic, policy, technology, advancement of science, and uncertain/little impact.

The category "advancement of science" was included because a number of researchers consider this in itself a positive social consequence, although earlier studies would have considered this as an "internal" factor rather than a social factor. As one respondent phrased it: "The quest for and sharing of knowledge are in and of themselves important social goals." (http://esi-topics.com/erf/2006/april06Rao_Turnshek.html). The category "uncertain about implications" applies to respondents who were unsure if their work had social/political implications, but then went on to list some possible outcomes. For example a respondent stated: "There may be no direct social or political implications of this research. However, it results in a better understanding of a climate system." (http://esi-topics.com/fbp/2005/december05CMontegut.html).

Health implications were an obvious and highly prevalent category. For example: "The control of pandemic influenza is not just a scientific concern." (http://esitopics.com/fmf/2006/january06-YiGuan.html). In another context, health impacts were also seen as contributing to the public understanding of science: "The paper can contribute to the ongoing debate over the use of human embryonic stem cells." (http://esi-topics.com/nhp/2005/november-05-YuvalDor.html). Philosophical and religious implications were also prominent. As an astronomer put it: "For some intellectually curious people, knowledge about other worlds is profoundly important, shaping their philosophy and religion and providing context for the precious value of our world." (http://esi-topics.com/nhp/2006/july-06-DebraAFischer.html). 
Table 1. Typology of social/political implications used to code author responses. The categories were created after a reading of the survey responses, and organized into main headings and subheadings

Typology of social and political implications

- General impact/benefit

- Health, general

$\begin{array}{ll}\circ & \text { Drug development } \\ \circ & \text { Mental health } \\ \circ & \text { Health, prevention of disease \& treatment } \\ \circ & \text { Health threat/risk } \\ \circ & \text { Food/nutrition }\end{array}$

- $\quad$ Environment, general

$$
\begin{array}{ll}
\circ & \text { Climate } \\
\circ & \text { Pollution }
\end{array}
$$

- Economic, general

$\begin{array}{ll}\circ & \text { Economic benefits } \\ \circ & \text { Economic inequality/burden }\end{array}$

- Policy, general

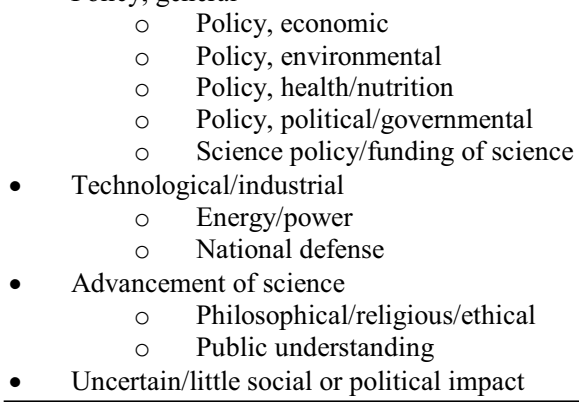

Finally, economic and technological implications, which were coded separately, are exemplified by the following excerpt: "The development of organic-based electronic devices may have strong implications for how the industry for electronics is organized." (http://esi-topics.com/erf/2005/december05-GillesHorowitz.html). In general, we can say that these and other responses show how intimately scientists' perceptions of social implications of science are tied with specific scientific findings and results.

The above categories are also clearly not mutually exclusive. Health issues can go hand in hand with policy, and can have implications for the economy. Therefore multiple categories may apply to any given response, and in fact the average response was classified in 3.2 categories.

Two individuals independently classified all responses into the categories, and the inter-rater reliability was computed using two widely accepted formulas - Cohen's Kappa and Perreault and Leigh's Reliability Index - both of which gave an agreement above $90 \%$. Figure 2 shows the frequency with which the various categories of social/political implication occur in the survey responses, based on the merged results of the two coders. The health treatment category is clearly the most prominent social 
implication comprising about $12 \%$ of all assignments. Second in frequency at $9 \%$ is the advancement of science, which some might not consider a social implication at all, but was clearly seen as such by the scientists responding to our survey. The third most frequent category was "no response" at 6 percent. Combining advancement of science with the "no response" and "uncertain" categories, gives about 22 percent of responses representing little or no social impact. Technological/industrial advance is fourth in occurrences which fits the conventional view of science as leading to technological applications.

To simplify the picture further, we can collapse the implication categories according to the main headings in Table 1, and re-compute the frequencies as shown in Table 2. This analysis reveals an even more dominant position of health implications at about $23 \%$. This number is in line with the MORI study which found that $22 \%$ of scientists gave the social and ethical implication of their research "to cure, treat or understand human illness or disease."

Table 2. Frequency of broad social/political implications coded in the author responses and counted at the level of the main headings in the typology of Table 1

\begin{tabular}{lc}
\hline Broad implication & Responses \\
\hline Health & 86 \\
No Response & 61 \\
Adv. science & 60 \\
Policy & 53 \\
Economic & 43 \\
Technology & 38 \\
Environment & 27 \\
\hline
\end{tabular}

One might ask whether the predominance of the health implications is expected given the number of papers from medical fields which would more or less automatically be considered as having social implications for health. Interestingly, about half of respondents to the full survey in clinical medicine and immunology gave no response to the question, despite the seemingly obvious impacts of their research.

First, we can collapse the fields of science shown in Figure 1 into broader areas, similar to our collapsing of the type of implications. For example, to create a broad health science category we have collapsed the fields of clinical medicine, immunology, neuroscience, pharmacology, and psychiatry/psychology. Such a rough aggregation, although far from ideal, allows us to examine more easily how responses are distributed across major fields and social implications. Table 3 gives a cross tabulation of broad implications versus fields of science, and we can see that while health as a social implication stands at about $23 \%$ of responses, health science papers comprise only about $18 \%$ of the papers. As clearly seen in the table, the health implication is invoked 
by scientists in a range of fields outside of medicine proper, in particular biological and physical sciences, and is disproportionate in its representation in number of papers.

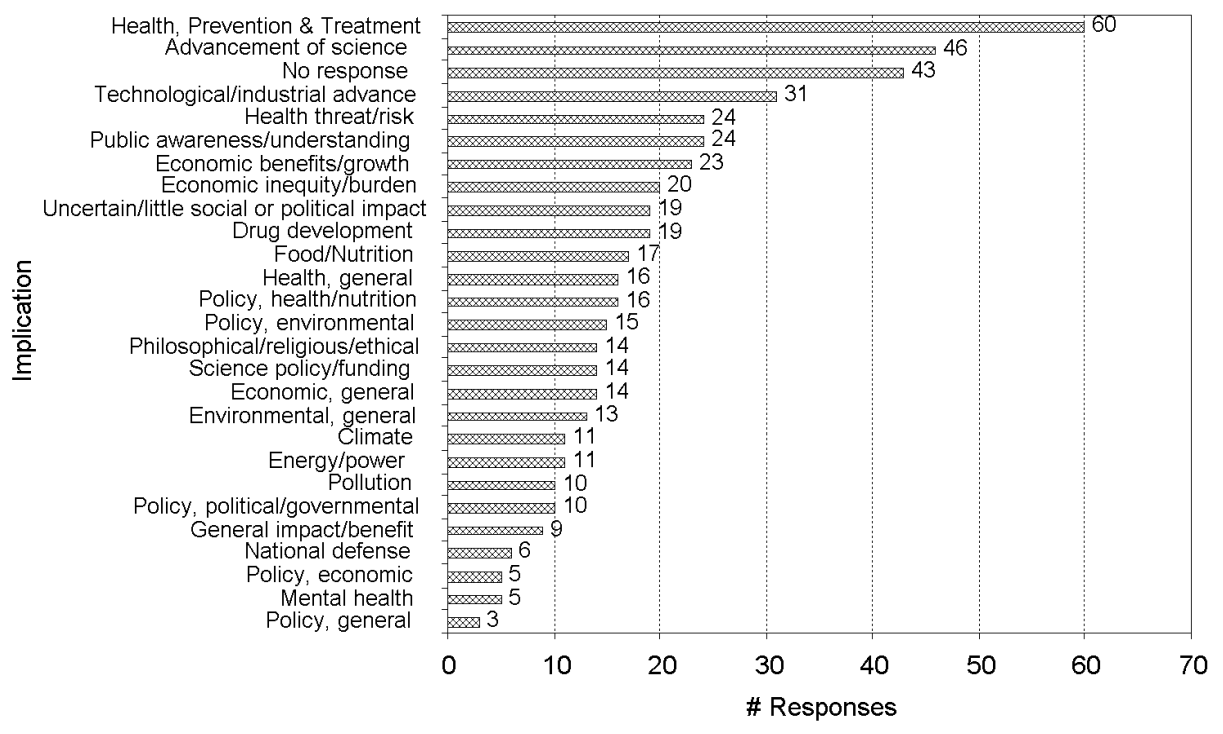

Figure 2. Frequency of social/political implications found in author responses ranked by frequency

One area of disagreement with MORI is their finding that a higher percentage of biomedical scientists say their research has social implications than do non-biomedical scientists $(79 \%$ versus $60 \%)$. Our results suggest that a slightly higher percentage of non-biomedical scientists cite social/political implications than do biomedical scientists (79\% versus $76 \%$ ). This discrepancy may relate to MORI's focus on social/ethical versus our social/political implications. Alternatively, it may be due to a difference in response rates for the two groups, or a greater desire on the part of non-biomedical scientists to discuss social implications or a greater reluctance on the part of biomedical scientists to do so.

Another factor that can perhaps shed light on the degree of motivation of different groups to discuss the social implications of research is the length of the response to the question. Measuring length of response by the average word count, we find that the longest responses were received from researchers giving environmental pollution as the social/political implication with an average of 157 words, followed by the health/nutrition policy implication with 134 words (Figure 3). Food/nutrition and environmental policy, areas that clearly have important public dissemination functions, had the next longest responses, suggesting a clear tendency for environmental and 
nutrition issues to elicit the more extended discussions. Summarizing the response length data for the scientific field and aggregating fields into four main branches (Table 4), we find that social scientists give the longest responses (132 words on average), followed by biological scientists, physical scientists, and health scientists with the shortest responses.

Table 3. Co-occurrence of broad implications with the discipline of the highly cited paper.

Implications were aggregated to the main headings and disciplines of science were aggregated into major fields

\begin{tabular}{|c|c|c|c|c|c|c|c|c|}
\hline Implication & Field & Bio Sci & Health Sci & Multidiscip & Physical Sci & Soc Sci & Total & Percent \\
\hline Economic & & 7 & 8 & 0 & 22 & 6 & 43 & $12 \%$ \\
\hline Environment & & 8 & 0 & 0 & 19 & 0 & 27 & $7 \%$ \\
\hline Health & & 32 & 31 & 1 & 18 & 4 & 86 & $23 \%$ \\
\hline No response & & 19 & 14 & 2 & 26 & 0 & 61 & $17 \%$ \\
\hline Policy & & 18 & 6 & 3 & 14 & 12 & 53 & $14 \%$ \\
\hline Adv science & & 20 & 7 & 3 & 24 & 6 & 60 & $16 \%$ \\
\hline Technology & & 6 & 1 & 0 & 29 & 2 & 38 & $10 \%$ \\
\hline Total & & 110 & 67 & 9 & 152 & 30 & 368 & \\
\hline Percent & & $30 \%$ & $18 \%$ & $2 \%$ & $41 \%$ & $8 \%$ & & \\
\hline
\end{tabular}

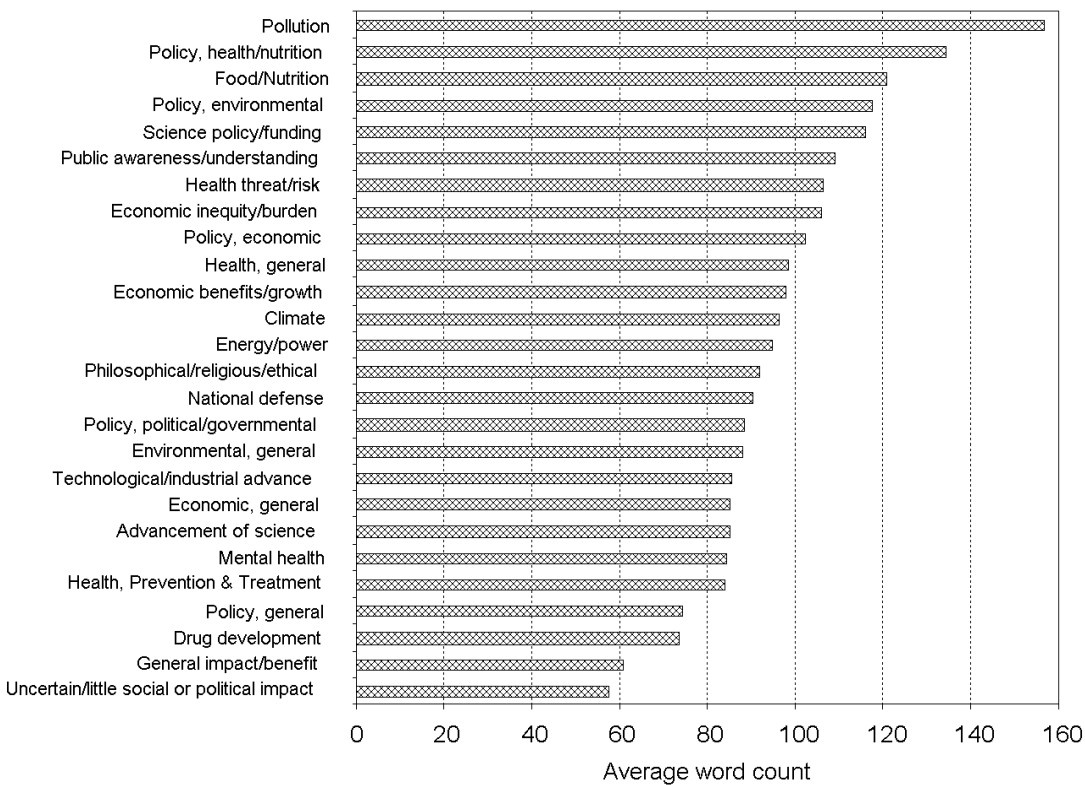

Figure 3. Average word count of response by the social/political implication 
H. SMALL \& AL.: Scientists' perceptions of the social and political implications

Table 4. Average word count of the responses aggregated by the broad scientific field of the papers

\begin{tabular}{lc}
\hline \multicolumn{1}{c}{ Broad field } & Average word count \\
\hline Social Science & 132 \\
Biological Science & 87 \\
Physical Science & 79 \\
Health Science & 73 \\
\hline
\end{tabular}

\section{Relation of social/political implications to topics and fields}

The cosine measure of association can be used to assess the strength of connection between scientific fields and the social/political implications (Table 5). The strongest of these associations shown in Table 5 are not unexpected due to the similarity of the field and the type of implication. For example, papers in the field of economics and business are strongly associated with economic policy, and materials science is associated with technological advance. More interesting are less predictable associations such as social science with health policy, environment/ecology with public awareness/understanding, and space science with the philosophical/religious/ethical implications.

Table 5. Association of selected implications with fields. The associations are computed as cosines, i.e., the co-occurrence of implication and field is divided by the square root of the product of the field and implication occurrences. Only associations with cosines having a value of 0.35 or greater are listed

\begin{tabular}{llc}
\hline Field & Implication & Cosine \\
\hline Economics \& Business & Policy, economic & 0.79 \\
Geosciences & Climate & 0.70 \\
Psychiatry/Psychology & Mental health & 0.63 \\
Agricultural Sciences & Food/Nutrition & 0.56 \\
Social Sciences, general & Policy, health/nutrition & 0.41 \\
Materials Science & Technological/industrial advance & 0.40 \\
Geosciences & Policy, general & 0.38 \\
Environment/Ecology & Policy, environmental & 0.37 \\
Environment/Ecology & Public awareness/understanding & 0.36 \\
Space Science & Philosophical/religious/ethical & 0.36 \\
\hline
\end{tabular}

Table 6. Fields of science of papers most strongly associated with the health implication given by respondents. Only those fields having a cosine association equal or greater than 0.1 are listed

\begin{tabular}{lc}
\hline Field & Cosine \\
\hline Microbiology & 0.31 \\
Neuroscience \& Behavior & 0.26 \\
Immunology & 0.25 \\
Pharmacology \& Toxicology & 0.23 \\
Mathematics & 0.22 \\
Clinical Medicine & 0.20 \\
Molecular Biology \& Genetics & 0.17 \\
Agricultural Sciences & 0.17 \\
Biology \& Biochemistry & 0.15 \\
Computer Science & 0.14 \\
Psychiatry/Psychology & 0.14 \\
Social Sciences, general & 0.11 \\
\hline
\end{tabular}


Table 7. Social/political implications most strongly associated with papers in the field of physics. Only those fields having a cosine association equal or greater than 0.1 are listed

\begin{tabular}{lc}
\hline Implication & Cosine \\
\hline Technological/industrial advance & 0.25 \\
Economic, general & 0.19 \\
Advancement of science & 0.16 \\
Economic benefits/growth & 0.15 \\
Energy/power & 0.11 \\
\hline
\end{tabular}

Such relationships can be further explored by selecting a specific implication and seeing what fields are strongly associated with it. For example, the health implication (Table 6) is not unexpectedly associated with the fields of microbiology, immunology, neuroscience, etc. More surprising are its weaker associations with the fields of mathematics, agriculture, computer science, and social sciences, again showing the dispersion of health issues across various fields.

Alternatively, we can examine a field of science and see what social/political implications are associated with it. For example, selecting physics as a field (Table 7), the three strongest associated implications are technological/industrial advance, economics, and advancement of science. Weaker associations are with economic benefit and energy technology.

We can also investigate at the level of individual topics and examine their patterns of implications. For the most prevalent topic in the sample, genomics, Table 8 shows the associated implications ranked by frequency. The less frequently associated implications such as drug development, technological/industrial advance, and public awareness/understanding, point to emerging issues for this topic.

The most frequent implications for nanotechnology (Table 9) suggest a greater emphasis on economic benefit and technology, but the environmental implication further down on the list points to an emerging and perhaps critical issue for this topic.

Similar analyses reveal the close coupling of stem cell research with the social issue of public understanding. Hence, the study of social implications at the topic level can reveal specific concerns that are perhaps masked at the field or disciplinary level, but may be of special significance for emerging areas of research.

Table 8. Social/political implications coded for survey responses associated with papers on genomics

\begin{tabular}{lc}
\hline Implication & Responses \\
\hline Health, Prevention \& Treatment & 13 \\
Advancement of science & 12 \\
Drug development & 5 \\
Health, general & 4 \\
Uncertain/little social or political impact & 3 \\
Technological/industrial advance & 3 \\
Public awareness/understanding & 3 \\
\hline
\end{tabular}


H. SMALL \& AL.: Scientists' perceptions of the social and political implications

Table 9. Social/political implications coded for survey responses associated with papers on nanotechnology

\begin{tabular}{lc}
\hline Implication & Responses \\
\hline Technological/industrial advance & 4 \\
Economic benefits/growth & 4 \\
Health, Prevention \& Treatment & 2 \\
Environmental, general & 2 \\
Energy/power & 2 \\
Advancement of science & 2 \\
\hline
\end{tabular}

\section{Mapping social and political implications}

Because multiple social/political implications have been assigned to a given response, it is possible to compute the co-occurrence of implications within responses, and create a map of the relationships between implications. The results of this exercise show how the implications group together to form larger aggregates (Figure 4).

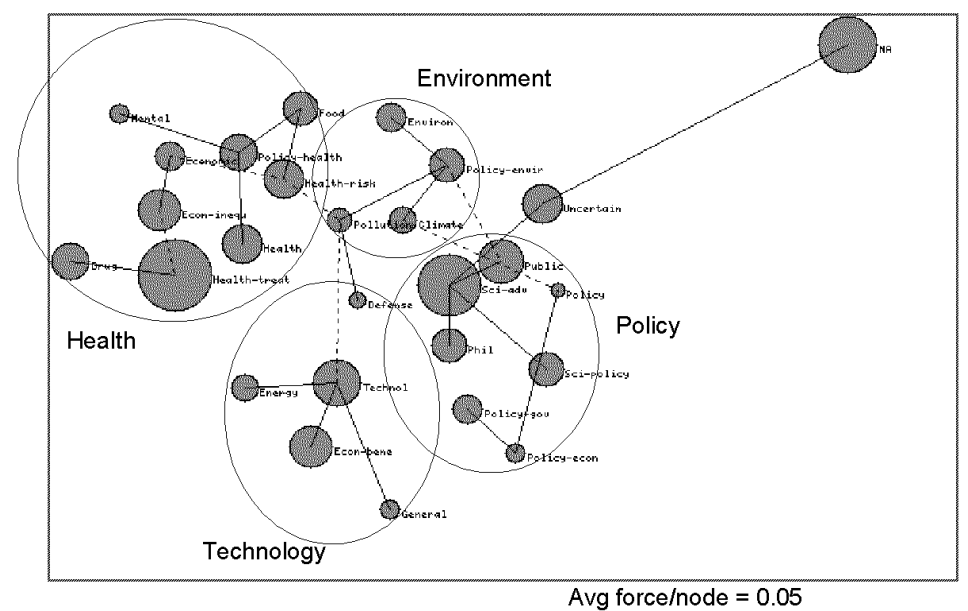

Figure 4. Map of social/political implications using the number of co-assignments of different implications to responses. The implication co-occurrences are normalized by the cosine formula and used proximities in a force placement algorithm. The average force per node represents the residual force after optimizing node positions

The structure obtained from a force-directed placement algorithm [SMALL, 2006] displays the strongest links as a solid line and weaker links as dotted lines. The force per node represents the average remaining residual force after the positions of nodes have been optimized by the force-placement algorithm. Circles have been drawn to 
show groupings of four clusters of implications labeled health, environment, policy, and technology. In addition there is a tail to the upper right of the map consisting of the nonresponses and the uncertain categories.

Interestingly, the policy cluster incorporates the scientific advance, public understanding, and philosophical implication categories along with other policy categories. The technology group includes energy, economic benefit, and defense. The environment group includes not surprisingly the environmental policy category along with climate and pollution. Finally the large health group incorporates almost all the categories that have a health dimension including health policy, two economic areas, and food. Note that the policy and economic categories are dispersed among the various groupings, policy categories appearing in three groupings, and economic categories in two. This structure suggests that the number of dimensions of social/political implications of science may in fact be fewer than the eight main categories in our original scheme (Table 1) and that four or five major headings might suffice.

\section{Conclusions}

This paper has explored an empirical approach to studying the social and political implications of science by gathering scientists' perceptions of the social impacts of their research output. It was found that 78 percent of scientists responding to a survey indicated that the research performed for their recent highly cited paper had such implications. Health related implications were the most common, but the number of papers in medical fields could not account for the abundance of health implications. Other non-medical fields of science perceived health implications for their research as well.

Our approach of gathering scientists' responses with respect to specific published work has the advantage of grounding their perceptions on actual research outcomes rather than gauging their general philosophical or theoretical views on how science might impact society. We are thus able to see how intimately such social and political consequences are tied to the details of scientific work, as for example, the impact of organic electronics on the electronics industry, and the impact of a possible pandemic influenza on world health. It is of course possible that scientists, when asked, would think of social impacts that perhaps would not have occurred to them in the normal course of their work, or which played no role in motivating or shaping that work. Some scientists did respond in a tentative and uncertain way to our question, as if perhaps such factors were not important to the framing of their research. Nevertheless, "no response" and "uncertain" responses only comprised about one-quarter of this sample.

The category of "advancement of science" captured what in earlier research on social consequences of science has been termed an "internal" or "intrinsic" criterion for evaluating scientific outcomes, rather than a social or "extrinsic" factor. 
The prominence of this category demonstrates the fact that many scientists believe that simply doing science and advancing knowledge is an important social outcome which is perhaps not separable in the scientists' minds from extrinsic social impacts.

When we break down our responders by field we find only a slight difference between physical and biomedical sciences in their tendency to cite social or political implications; all social scientists reported at least one such implication. If we use the length of response to gauge the depth of commitment to such issues, we find that topics with a strong component of public dissemination have the longest responses, but we found no strong disciplinary associations for length of response. Field specific preferences for certain types of social/political implications are however apparent, for example, social scientists with health policy, environmental scientists with public awareness/understanding, and space scientists with philosophical implications. However, fields of science were not found to group strongly according to the social/political implications they shared. That is, physical sciences or life sciences do not tend to cluster around distinct sets of common implications, but rather loosely associate through many different implications.

The analysis of social implications at the topic, rather than discipline, level revealed topic specific concerns such as nanotechnologies with environmental impacts, or stem cells with public understanding. Such an analysis opens up the possibility of using this method in a systematic way to track the social and political issues surrounding a specific emerging area of science.

The traditional assumption that the social implications of research are determined primarily by whether the research is applied or mission oriented rather than basic or pure did not seem to have much bearing on our data. Our results show that some scientists whose work would traditionally be considered applied, such as clinical research or computer science, did not report social/political implications, while others working on what is commonly considered basic research provided some surprising connections with the wider public such as relevance to public understanding, policy debates, and economic payoff.

The structural analysis of implication co-occurrences that resulted from our coding, showed a tendency of certain implication types to cluster or group on the map, suggesting that a more simplified categorization might by justified. The fact that policy and economic related categories were somewhat dispersed among the four major groups - environment, policy, technology, and health - suggests that specialized forms of these categories, for example, health policy and health economics, might more properly be incorporated in the larger health dimension. If we were to use this mapping approach with survey responses for a specific scientific topic, it seems likely that we would be able to create a map of social implications at the level of the research topic, which might give us a new tool for communicating the value of research to a policy or administrative audience. 
Future studies might also examine the extent to which the social and political implications of research cited by our respondents are mirrored in the policy and governmental discussions regarding the funding of science in specific areas. Although Weinberg states that such considerations may only apply to big science projects, it seems to us that a broad view of the types of social impacts of research as conceived by scientists themselves regarding their actual work and sampled across many fields has a role to play in the rationale for public support of both large and small projects.

\section{References}

BARNS, I. J., WILSON, S. A. (1996). Scientists and the broader community. International Journal of Environment and Pollution, 6 (2-3) : 142-148.

Bernal, J.D. (1965), Science in History, Vol. 3, The Natural Sciences in Our Time. (Cambridge, Mass.: MIT Press).

Brooks, H. (1968), Government of Science. (Cambridge, Mass.: MIT Press), Chapter 3.

COHEN, J. (1968), Weighted kappa: Nominal scale agreement with provision for scale disagreement or partial credit. Psychological Bulletin, 70 : 213-220.

Corrado, M., Pooni, K., Hartfree, Y. (2000), Role of Scientists in Public Debate. Report for the Wellcome Trust by Market and Opinion Research International (MORI). (London: The Wellcome Trust).

GARFIELD, E. (1981), Citation Classics: four years of the human side of science. Current Contents, 22 (1 June 1981) : 5-16. Reprinted in: Essays of an Information Scientist, Vol. 5 (Philadelphia: ISI Press), pp. $123-134$

Murphy, K., TOPEL, R. (2003), Diminishing returns? The costs and benefits of improving health. Perspectives in Biology and Medicine, 46 (3) : S108 - S128.

Narin, F., Hamilton, K. S., Olivastro, D. (1997), The increasing linkage between U.S. technology and public science. Research Policy, 26 (3) : 317-330.

NATIONAL SCIENCE BOARD (1993), Science \& Engineering Indicators - 1993. Washington, DC: U.S. Government Printing Office, Chapter 7.

Perreault, W. D., LeIGH, L. E. (1989), Reliability of nominal data based on qualitative judgments. Journal of Marketing Research, 26 (2) : 135-148.

Price, D. J. DE Solla (1976), Extrinsic value theory for basic and applied research. Policy Studies Journal, $5(2): 160-168$.

SMALl, H. (2004), Why authors think their papers are highly cited. Scientometrics, 60 (3) : $305-316$.

SMALL, H. (2006), Tracking and predicting growth areas in science. Scientometrics, 68 (3) : $595-610$.

WeINBERG, A. M. (1963), Criteria for scientific choice. Minerva, 1 (2) : $159-171$. 